\title{
EFFECT OF MULTIPATH ROUTING IN AUTONOMOUS MOBILE MESH NETWORKS
}

\author{
Sangheethaa $\mathbf{S}^{\mathbf{1}}$, Neethu Janardhanan ${ }^{2}$ \\ ${ }^{1}$ Assistant Professor, Dept. of Electrical and Computer Engg, School of Engineering and Technology, Dilla \\ University, Dilla, Ethiopia \\ ${ }^{2} P G$ Scholar, Dept of Computer Science, Vimal Jyothi Engineering, Kannur University, Kerala, India
}

\begin{abstract}
Autonomous mobile mesh networks are a combination of mobile ad hoc networks and mesh networks. Mobile ad hoc networks are temporarily formed for a specific purpose, without any fixed infrastructure. Mesh networks are fully connected networks with the support of fixed infrastructure. Autonomous mobile mesh networks (AMMNET in short) [1], is the framework designed for supporting mesh networks, which are mobile still expects connectivity as opposed to ad hoc networks. This paper proposes a multipath routing technology for AMMNETs which provides better packet delivery ratio in AMMNETs. The simulations are done in ns2. The results prove that, multipath routing technology works well with AMMNET framework.
\end{abstract}

Keywords: AMMNET, routing, multipath, ns2.

\section{INTRODUCTION}

Ad hoc networks [2] are temporary wireless networks normally formed at emergency situations where there are no centralized authorities like base station or access points. Ad hoc networks have special characteristics. The routers are free to move randomly and organize themselves arbitrarily; thus, the network's wireless topology may change rapidly and unpredictably. Ad Hoc networks are used for creating temporary networks. The main application areas of Ad-hoc networks are; Creation of instant infrastructure, Disaster relief, network connection to remote areas where installation of base station is not feasible. An ad hoc network consists of nodes which are free to move. The nodes may be anywhere, in airplane, ships, trucks, cars even on every people.

One important challenge in MANETs is to manage the network partitions due to dynamic topology. This problem is addressed in the paper [2], and they have proposed a framework called as AMMNET- autonomous mobile mesh networks. In a standard mesh network, mesh nodes may be mobile, but the mesh routers are stationary. In AMMNET, the routers will move along with mobile mesh clients to serve them in communication. We have conducted simulations and understood that, when AMMNET is used with multipath routing, better throughput and packet delivery ratio can be achieved. In [2] the authors have used AODV [3] . This paper is organized in to following sections. Next section briefs some of the existing approaches related to this paper. Section 3 discusses the AMMNET model in detail. Section 4 explains the proposed multipath routing in AMMNET. Section 5 gives simulation results and finally, section 6 concludes the paper along with some future research scopes.

\section{RELATED WORK}

Some literatures [4 ] [5] study on improving the network topology in wireless mesh networks. But our paper deals with mobile mesh networks where routers also moving. Wireless sensor covering is another area related to our paper. But in sensor networks, tracking of sensors become easy because the deployment of sensors is fixed, and location of them is already known. There is no much dynamicity to be considered while tracking sensors. But mobile mesh nodes will move dynamically and tracking them is more challenging than sensor nodes. Localization techniques [6],[7], [8], [9] are another area where mobile clients' location is tracked. But these are techniques to exactly know where a client is. But our paper need just whether mobile client is there are not, it does not need the exact location of clients.

\section{AMMNET}

Autonomous mobile mesh network is the framework for forwarding data between clients in a mobile mesh networks. In normal mesh networks, routers will be stationary. But in AMMNET, routers will have to move along with the mobile mesh nodes in order to provide them seamless connectivity. Mesh nodes are free to move, they broadcast beacon messages periodically. On receiving this beacon messages, the mesh router can detect presence of a mobile mesh node. This framework assumes group mobility patterns among the mobile clients. When a group leader moves in a particular direction, all the clients in that group also starts moving in that direction. Three types of mobile mesh nodes are defined in [1] based on their rolls. Intra group routers, intergroup routers and free routers. A mesh node is an intra group router, if it could serve at least one mobile client by detecting its beacon message. These are responsible for communication and monitoring inside a particular group. 
Inter group router as the name suggest, are responsible for communication between the groups. A node is called a free router if it not intra group or inter group router.

Three algorithms are proposed in [1] to decide which node will become intra, which will be inter and which one will be a free router. Based on these algorithms, a free router may become a intra group router, or inter and vice versa. The main function of these routers will be to keep track of the mobile clients in its range. When a mobile clients' beacon is missing at a particular time, the router has to take necessary action to detect that moving client and make arrangements for that client to be in the connected area. [1] also proposes some optimization techniques to decide the number of intra and inter group routers active at a time. Interested readers can know more about these algorithms from [1].

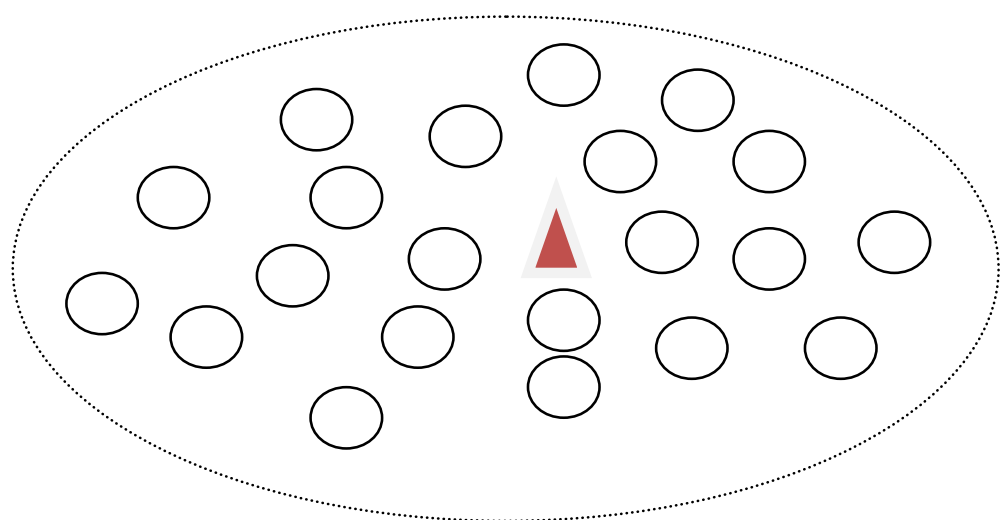

Fig 3.1 Initial network with one intra group router

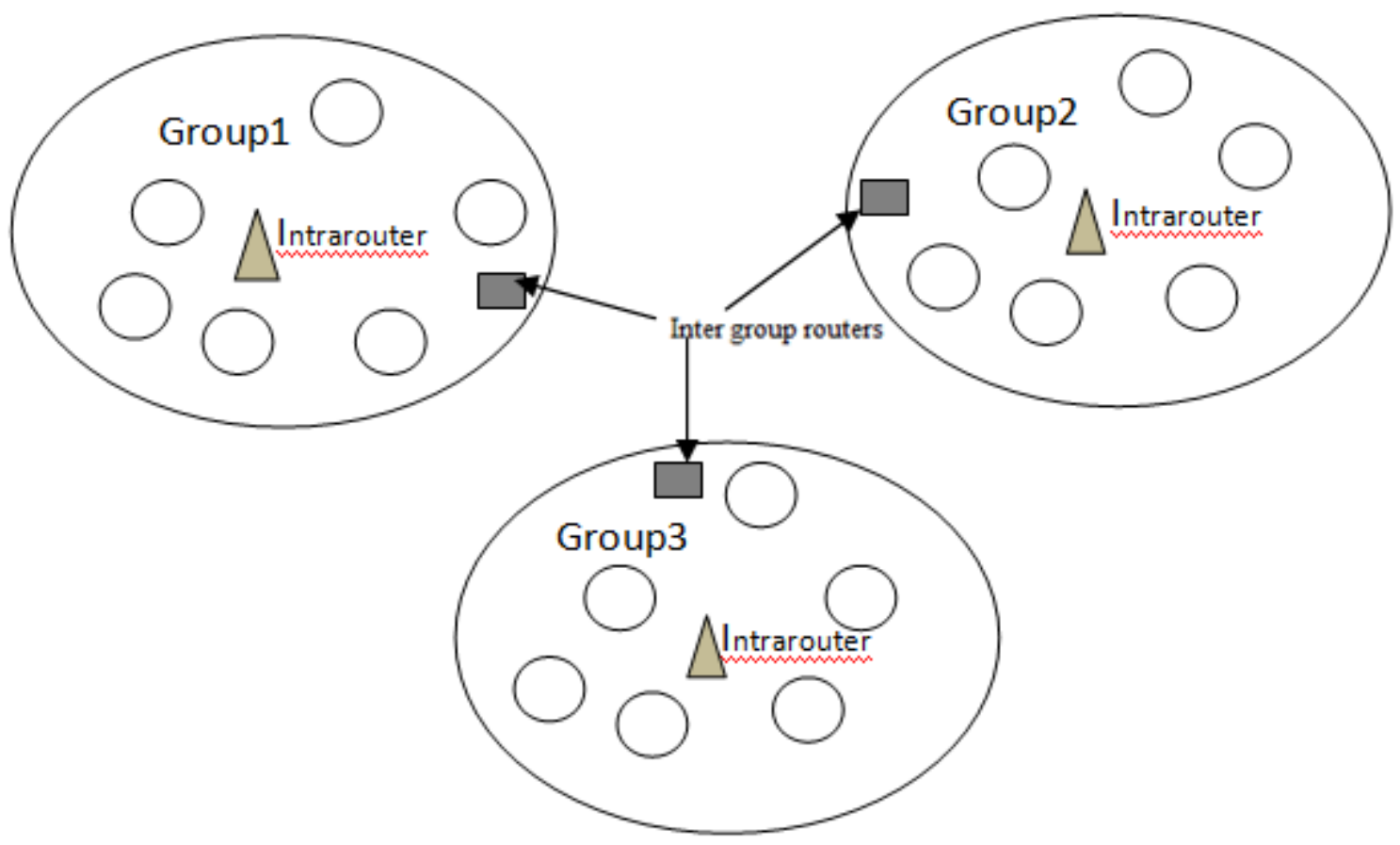

Fig 3.2 Network with 3 groups of nodes

The figure 3.1 shows an example for network with some number of nodes. This is the initial network, where all nodes start from the same location and one intra group router is enough to support the entire network. The intra group router is shown as a rectangle. The radio range of this router is shown as the outer big circle. This means that entire network is under one group.
Figure 3.2 shows the network with three groups. Each group has a intra group router to help communication within a group. Communication between groups is possible with the help of inter group routers.

[1] deals intensively in how to track a mobile client. It is not concentrating on increasing the throughput or increasing the packet delivery ratio. It clearly mentions that the main objective is to keep track of the clients, rather than thinking 
whether data is delivered to correct destination or not. But the ultimate aim of tracking should be communication. AMMNET framework does not guarantee delivery of packets to the correct destination. So this paper utilizes the multipath routing concept to ensure that data is delivered to the destination and thus increasing the throughput and packet delivery ratio of AMMNET. Next section explains the concept of multipath routing and its use in AMMNET.

\section{MULTIPATH ROUTING IN AMMNET}

Multipath routing [2] means routing the packets through different available routes to the destination. Existing ondemand protocols like AODV, DSR are giving shortest path to the destination. They use route request message to find a route. Route request is sent as a broadcast message by the source which wants to send data. Intermediate nodes and/or destination will give route reply. On receiving the first route reply, or best route reply, the source starts transmission of data. The source is actually receiving multiple route replies from the intermediate nodes as well as from the destination. So it would be better, if more than one route is used for transmission of data to the destination.

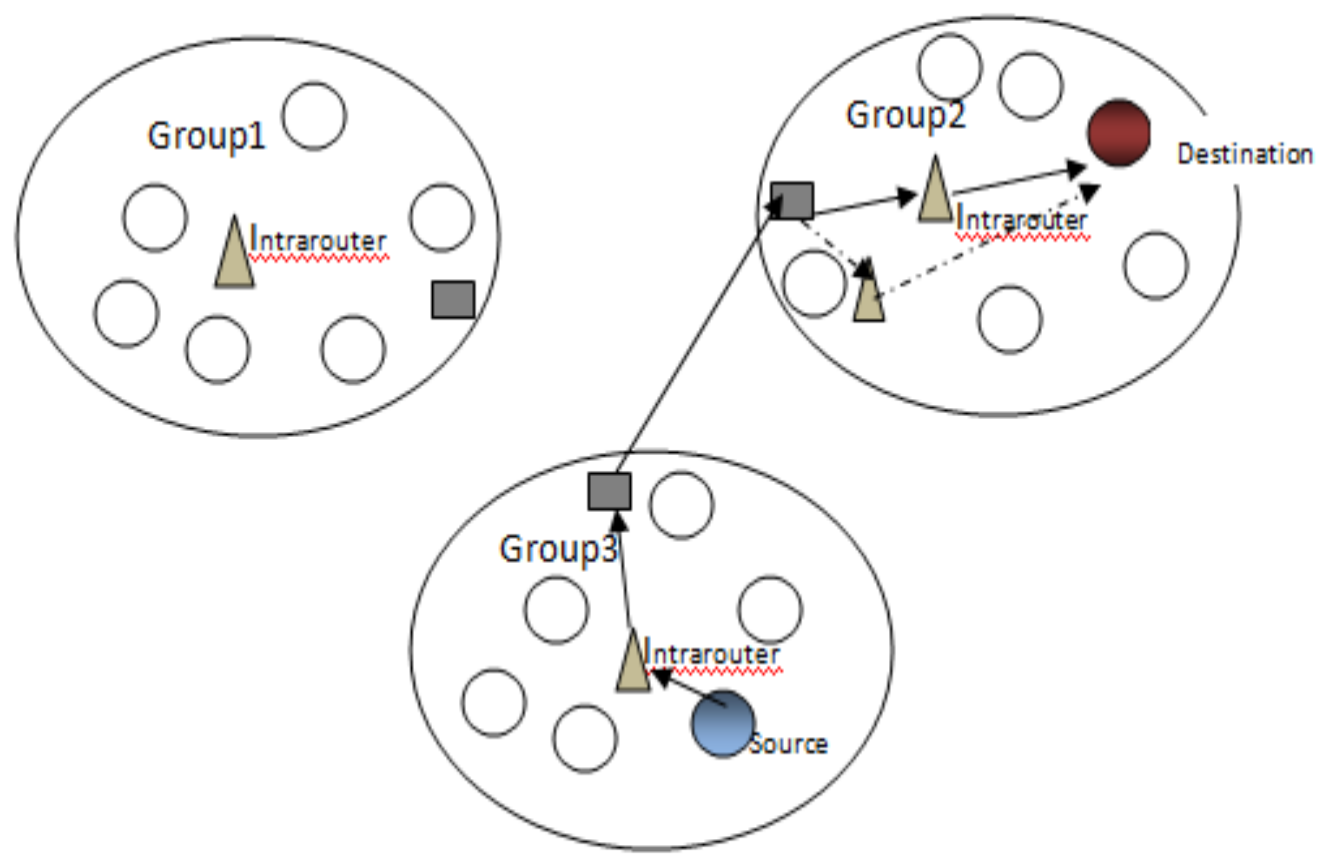

Fig. 4.1: Example multiple paths to destination from source

AOMDV [10] is a multipath routing version of the Adhoc on-demand routing protocol (AODV). It is proved in [10] that AOMDV is superior to AODV and other existing routing protocols. It utilizes all alternative paths, thus not monopolizing any routes. It ensures delivery

of packets to the correct destination. Figure 5.1 gives an example for two alternative paths to reach destination in group 2 from a source in group 3 through different intra group routers. Even though multiple paths are not available outside the group, i.e the routes are through the same intergroup routers, still inside a group, multiple paths are available.

\section{SIMULATION RESULTS}

Simulations are done in ns2 [11] version 2.35. The parameters set for simulation are given in table 5.1. The parameters used for performance comparison are, Packet delivery ratio and Throughput.
Table 5.1 Simulation parameters

\begin{tabular}{|l|l|l|}
\hline S.No & Parameter & Values \\
\hline 01 & Number of nodes & 39 \\
\hline 02 & Speed of nodes & $15 \mathrm{~m} / \mathrm{s}$ \\
\hline 03 & Simulation time & $100 \mathrm{~s}$ \\
\hline 04 & Initial Energy & 500 joules \\
\hline 05 & Type of traffic & UDP \\
\hline 06 & Number of connections & 12 \\
\hline
\end{tabular}

Figure 5.1 shows the packet delivery ratio achieved for AMMNET and AMMNET with multi-path routing. From the graph it is clear that, using multipath routing in AMMNET increases the packet delivery ratio by $20 \%$. When the traffic increases, AMMNET is not able to achieve better packet delivery ratio, where as in AMMNET with multi path routing, PDR increases. This is due to the fact that data is transmitted over multiple available paths. Note that AMMNET uses AODV for updating its routing table. But multipath routing uses AOMDV. 


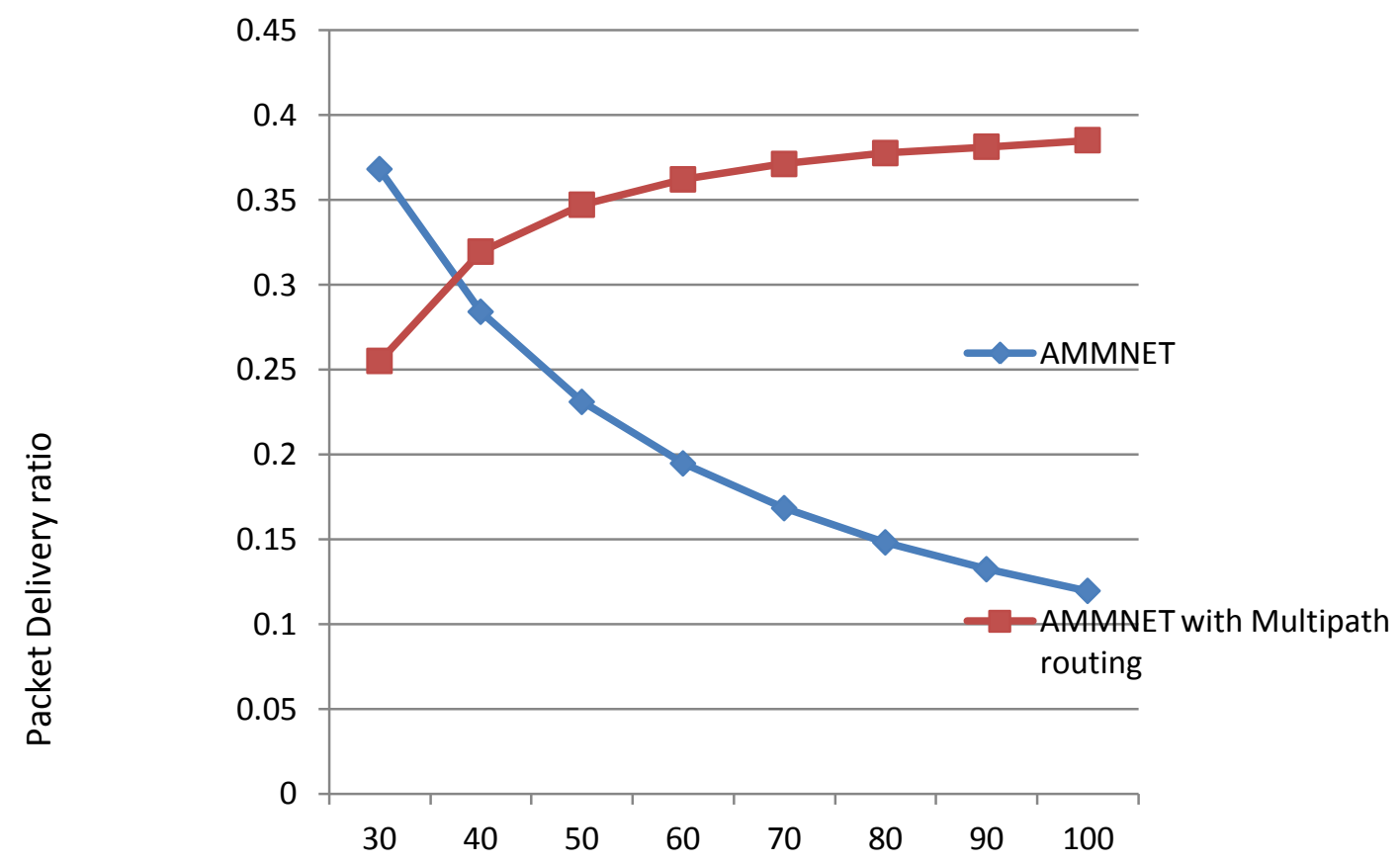

Simulation time in seconds

Fig 5.1 Packet Delivery ratio Vs. Simulation Time

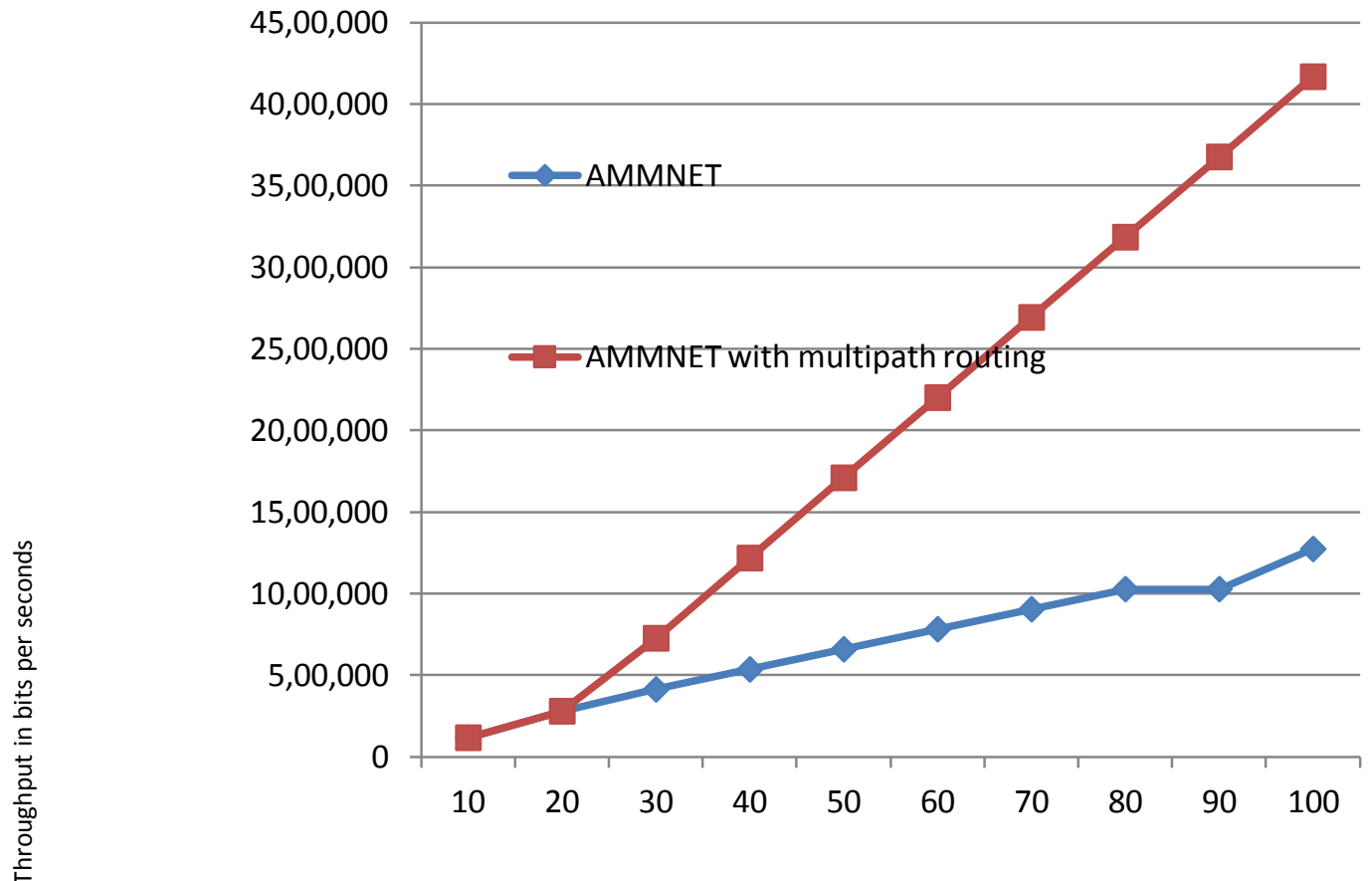

Simulation time in seconds

Fig 5.2 Throughput Vs. Simulation Time 
Figure 5.2 shows that the throughput is increased by $40 \%$ in AMMNET with multipath routing, compared to AMMNET. It is also due to data transmission over multiple available paths. Thus from the graphs it is clear that, AMMNET when used with multi path routing technology, improves PDR, throughput and efficiency.

\section{CONCLUSION}

This paper has added multipath routing concept along with AMMNET and proved that, using multipath routing in AMMNET will increase the throughput as well as packet delivery ratio. The simulations are done for just 39 nodes, In future, the number of nodes can be increased and the effect of multipath routing in AMMNET can be analyzed.

\section{REFERENCES}

[1]. Wei-Liang Shen, Chung-Shiuan Chen,Kate Ching-Ju Lin, "Autonomous Mobile Mesh Networks" IEEE TRANSACTIONS ON MOBILE COMPUTING, VOL. 13, NO. 2, FEBRUARY 2014

[2]. http://en.wikipedia.org/wiki/Wireless_ad_hoc_network.

[3]. C. E. Perkins and E. M. Royer, "Ad hoc On-Demand Distance Vector (AODV) routing," RFC 3561, Jul. 2003. [Online]. Available: http://www.ietf.org/rfc/rfc3561.txt

[4]. J. Tang, G. Xue, and W. Zhang, "Interference-Aware Topology Control and QoS Routing in Multi-Channel Wireless Mesh Networks," Proc. ACM MobiHoc, 2005.

[5]. A. Naveed, S. Kanhere, and S. Jha, "Topology Control and Channel Assignment in Multi-Radio Multi-Channel Wireless Mesh Networks," Proc. IEEE Int'l Conf. Mobile Adhoc and Sensor Systems (MASS), 2007.

[6]. A. Savvides, C.-C. Han, and M.B. Strivastava, "Dynamic Fine-Grained Localization in Ad-Hoc Networks of Sensors," Proc. ACM MobiCom, 2001.

[7]. C. Savarese, J.M. Rabaey, and K. Langendoen, "Robust PositioningAlgorithms for Distributed Ad-Hoc Wireless Sensor Networks,"Proc. USENIX Ann. Technical Conf., 2002.

[8]. J. Liu, Y. Zhang, and F. Zhao, "Robust Distributed Node Localization with Error Management," Proc. ACM MobiHoc, 2006.

[9]. H.T. Kung, C.-K. Lin, T.-H. Lin, and D. Vlah, "Localization with Snap-Inducing Shaped Residuals (SISR): Coping with Errors in Measurement," Proc. ACM MobiCom, 2009.

[10]. Mahesh K. Marina,Samir R. Das“ Ad hoc on-demand multipath distance vector routing" in WIRELESS COMMUNICATIONS AND MOBILE COMPUTING Wirel. Commun. Mob. Comput.2006;6 :969-988 Published online in Wiley InterScience (www.interscience.wiley.com). DOI: $10.1002 /$ wcm.432

[11]. Ns2- www.isi.edu/nsnam/ns/ 